\title{
The preoperative myocardial perfusion and internal mammary artery graft blood flow relationship in patients with ischemic cardiomyopathy
}

\section{Sergey S. Gutor}

Cardiology Research Institute, Tomsk National Research Medical Centre, Russian Academy of Sciences

\section{Sergey L. Andreev}

Cardiology research institute, Tomsk National Research Medical Centre, Russian Academy of Schiences

Vladimir V. Shipulin ( $\nabla$ shipartphoto@gmail.com )

Tomsk National Research Medical Center, Russian Academy of Sciences https://orcid.org/0000-00019887-8214

\section{Andrey V. Mochula}

Cardiology Research Institute, Tomsk National Research iMedical Centre, Russian Academy of Sciences

\section{Vasiliy V. Zatolokin}

Cardiology Research Institute, Tomsk National Research Medical Centre, Russian Avademy of Sciences

\section{Andrey S. Pryahin}

Cardiology Research Institute, Tomsk National Research Medical Centre, Russian Academy of Sciences

Vladimir M. Shipulin

Cardiology Research Institute, Tomsk National Research Medical Centre, Russian Academy of Schiences

Boris N. Kozlov

Cardiology Research Institute, Tomsk National Research Medical Centre, Russian Academy of Sciences Konstantin V. Zavadovsky

Cardiology Research Institute, Tomsk National Research Medical Centre, Russian Academy of Sciences

\section{Research Article}

Keywords: transit-time flow measurement, myocardial perfusion imaging, coronary artery bypass grafting, coronary artery disease, ischemic cardiomyopathy

Posted Date: February 4th, 2022

DOI: https://doi.org/10.21203/rs.3.rs-1316785/v1

License: (c) (1) This work is licensed under a Creative Commons Attribution 4.0 International License. Read Full License 


\section{Abstract}

Objective. The purpose of this study was to determine whether myocardial perfusion in stress/rest conditions estimated by SPECT can be used to predict transit time blood flow characteristics and early graft failure in left internal mammary artery (LIMA) to left anterior descending artery (LAD) grafts in patients with ischemic cardiomyopathy.

Methods. The study group consisted of 47 patients with ischemic cardiomyopathy. Intraoperative transittime flow measurement (TTFM) of LIMA to LAD grafts were performed in all patients. All patients were also examined with preoperative stress and rest myocardial perfusion SPECT with ${ }^{99 \mathrm{~m}} \mathrm{TC}-\mathrm{MIBI}$. Anastomotic patency was considered satisfactory with a normal waveform of blood flow, diastolicdominant blood filling, and a mean flow value greater than $11.5 \mathrm{ml} / \mathrm{min}$.

Results. All 47 patients with LIMA to LAD grafts were divided into two groups; those with graft satisfactory patency $(n=40)$ and those with unsatisfactory patency $(n=7)$ as determined by TTFM. We found differences among these groups in global summed rest score $(t=3.7, p=0.003)$, total perfusion deficit $(t=3.0, p=0.014)$, and LAD territory TPD $(t=2.4, p=0.04)$ characteristics in preoperative rest myocardial perfusion SPECT imaging. Additionally, the flow characteristics were different in patients with and without surgical left ventricle reconstruction $(U=153.5, p=0.012)$.

Conclusions. Preoperative myocardial perfusion characteristics have associations with blood flow in LIMA to LAD graft in patients with ischemic cardiomyopathy and LAD territory rest TPD have the potential to be a predictor of early graft failure.

\section{Introduction}

Coronary artery bypass grafting (CABG) is a widely used treatment for coronary artery disease (CAD), and it is mandatory for patients with ischemic cardiomyopathy (ICMP). While most chronic atherosclerotic narrowing of coronary arteries can be successfully bypassed, revascularization failures do happen $[1,2]$. Thus, the literature suggests that roughly $10 \%$ of all by-pass grafts occluded in the short-term and some of the authors call graft patency the "Achilles heel" of CABG $[3,4]$.

Graft patency is influenced by many factors including intramyocardial tension, graft conduits and design ( $\mathrm{Y}$ and $\mathrm{T}$ configuration), graft destination artery, and pacing modality and atrioventricular delay value [5].

Thus, internal mammary arteries (IMAs) are frequently used for CABG as the most reliable conduit. It is very crucial to select coronary artery for IMAs graft carefully because in some cases internal graft flow might be low by severe vasoconstriction or morphological state of the target artery myocardium territory and lead to graft failure in the end [6-8].

Until recently, there was not an "on-table" complete and user-friendly technique for graft patency assessment which would not drastically increase operation time. Transit-time flow measurement (TTFM), 
however, became such a method. Before the early 2000s, some scientists had questioned the capabilities of this method and then TTFM became the most common intraoperative small variable method of assessing graft function [9]. In 2010 it was included in the ESC (European Society of Cardiology) and EACTS (European Association for Cardio-Thoracic Surgery) Guidelines on myocardial revascularization (it is present in the version of 2018) [3, 10-15].

However, there is an ongoing dispute about the cut-point values for this method in modern literature and many researchers are trying to find cut-point value for each conduit type. For arterial conduits, $\mathrm{H}$. Oshima et al (2016), for example, set the mean flow (Q) value at $11.5 \mathrm{ml} / \mathrm{min}$ as the cut-off value between satisfactory and not-satisfactory grafts [1].

Despite recurring studies of the topic, L. Niclauss critical review (2017) suggests the influence of quantity (vessel territory distribution) and quality (myocardial morphological state) of the graft perfusion area on TTFM and conduits outcome have not included by anyone and should be studied [16]. In eComment to H. Oshima et al. article, N. Hudorovic suggest preoperative SPECT as useful to predict the regional functional recovery in the akinetic area after CABG, but only $29 \%$ of patients underwent preoperative SPECT in the study [1]. Moreover, routinely used preoperative SPECT with and without stress can provide doctors and medical scientists with vital information about coronary vessel perfusion area, including stable (most probably post-infarction scar) and unstable (ischemic) defects. This study investigated whether myocardial perfusion in stress and rest conditions estimated by SPECT can be used to predict blood flow characteristics and early graft failure in LIMA to LAD grafts in patients with ICMP.

\section{Materials And Methods}

\section{Study population}

The study group comprised of 47 consecutive patients (mean age $61.1 \pm 5.1$ years, $95.7 \%$ male) with ICMP, who underwent $C A B G$ with LIMA to LAD graft and left ventricle $(L V)$ reconstruction according to standard indications [17]. Within a week of surgery, stress and rest myocardial perfusion SPECT with ${ }^{99 \mathrm{~m}} \mathrm{Tc}-\mathrm{MIBI}$ and interoperation TTFM of LIMA grafts were performed on all patients.

Inclusion criteria were: 1) at least 3 months after acute myocardial infarction; 2) $\geq 75 \%$ stenosis of left main or proximal left anterior descending artery (LAD) or $\geq 75 \%$ stenosis of two or more major epicardial vessels; 3) left ventricular ejection fraction (EF) $\leq 40 \%$; 4) left ventricular end-systolic index $\geq 60 \mathrm{ml} / \mathrm{m} 2$ (by transthoracic echocardiography) [17].

Exclusion criteria were: 1) rheumatic or inflammatory heart disorders; 2) acute coronary syndrome; 3 ) recent (< 6 months) cerebral ischemic attack; 4 ) acute or chronic right ventricle failure (by transthoracic echocardiography); 5) severe pulmonary hypertension; 6) contraindications for cardiopulmonary bypass; 7) contraindication to adenosine administration. 
The study was approved by the Local Ethical Committee and conformed to the Declaration of Helsinki on Human Research. Written informed consent was obtained from each patient after explanation of the protocol, its aims, and potential risks.

\section{Surgical technique}

All operations were performed under conditions of norm-thermal cardiopulmonary bypass standard procedure. A great saphenous vein and a LIMA were harvested according to the routine procedure, the last one was wrapped with papaverine solution in surgical drape to prevent spasms [18].

In all cases, the LIMA was grafted to the left anterior descending artery and the great saphenous vein was sewn for other target vessels. No sequential anastomoses were included in this series. The LV reconstruction using the D. Cooley or V. Dor method with L. Menicanti modification was made in the case of an LV aneurysm based on preoperative examination and intraoperative evaluation $[19,20]$.

\section{Coronary angiography}

Quantitative coronary angiography was performed after catheterization of the femoral artery by the Seldinger's technique on the Axiom Artis coronary angiography system (Siemens; Erlangen, Germany) in a single scheme: a multi-projection right then left coronary angiography according to the method of $\mathrm{M}$. Judkins [21]. All coronary angiographies were done during the preoperative period.

\section{TTFM and evaluation criteria for anastomosis satisfactory}

Transit-time flow measurement was performed with VeriQ System (Medi-StimAS, Oslo, Norway) after all anastomoses, ventricle and valves reconstructions were completed, the heart-lung machine was disconnected and hemodynamic parameters became stable (mean arterial pressure fixed of 75-85 $\mathrm{mmHg})[3,22]$. Flow parameters recorded in this study included mean graft flow $(\mathrm{Q}, \mathrm{ml} / \mathrm{min})$, higher pulsatility index $(\mathrm{PI})$, and diastolic filling (DF, \%).

A Q value greater than $11.5 \mathrm{ml} / \mathrm{min}$, a normal waveform of blood flow, and diastolic-dominant blood filling was considered as a surrogate marker for satisfactory anastomotic patency [1].

\section{Myocardial perfusion imaging}

Patients were instructed to refrain from caffeine, substances containing methylxanthine, and to avoid nitrates, calcium channel blockers, and beta-blockers for at least $24 \mathrm{~h}$ before the scan. All scans were performed after overnight fasting.

All patients underwent a 2-day stress/rest protocol. A pharmacological stress-test (adenosine, 140 $\mathrm{mg} / \mathrm{kg} / \mathrm{min}$ for 6 minutes) combined with low-level exercise was performed in all patients [23]. The heart rate, systemic blood pressure, and 12-lead electrocardiogram were monitored before, during, and after the stress test. A dose of $370 \mathrm{MBq}$ of ${ }^{99 \mathrm{~m}} \mathrm{Tc}$-sestamibi was injected after 3 minutes of stress testing and the 
same dose on the next day for rest study like described in ASNC guidelines for SPECT nuclear cardiology procedures [23]. Pharmacologic stress testing did not lead to atria-ventricle (AV) conduction delay and/or to ST-segment depression in any patient. The total effective radiation dose was 6-7.3 mSv.

The SPECT data were acquired one hour after injection for both the rest and the stress studies with a solid-state detector CZT cardiac SPECT/CT system (GE Discovery NM/CT 570c). The acquisition time was 7 minutes. The myocardial perfusion imaging (MPI) scans were acquired using low energy multipinhole collimator and 19 stationary detectors which simultaneously imaged 19 different views without detector rotation. The acquisition matrix was $32 \times 32$ pixels (pixels sizes $4 \times 4 \times 4 \mathrm{~mm}$ ). Each detector contains $32 \times 32$ pixelated $(2.46 \times 2.46 \mathrm{~mm})$ CZT elements. A $20 \%$ energy window at $140 \mathrm{keV}$ was used. Patients were imaged in the supine position with arms placed over their heads.

CZT images were reconstructed on the dedicated workstation (Xeleris 4.0; GE Healthcare, Haifa, Israel) using maximum-penalized-likelihood iterative reconstruction (60 iterations; Green OSL Alpha 0.7; Green OSL Beta 0.3) to acquire perfusion images in standard cardiac axes (short axis, vertical long axis, and horizontal long axis). The software Myovation for Alcyone (GE Healthcare, Haifa, Israel) was used for image reconstruction, and Butterworth post-processing filter (frequency 0.37 ; order 7) was applied to the reconstructed slices. The reconstruction was performed in a $70 \times 70$ pixels matrix with 50 slices.

\section{MPI interpretation}

Raw MPI-CZT data at stress and rest were visually assessed for motion and attenuation artifacts. Stress/rest images were analyzed with a commercially available software package Cedars QGS/QPS (Cedars-Sinai Medical Center, Los Angeles, CA, USA).

Left ventricle myocardium was presented in a 17-segment polar map format and was computed separately for each vascular territory by the AHA guidelines [24]. Each of the 17 segments was scored based on a semi-quantitative 5-point scoring system: 0 - normal uptake; 1 - mild uptake reduction; 2 moderate uptake reduction; 3 - severe uptake reduction; and 4 - an absence of radiotracer.

Myocardium perfusion was assessed globally (Global) and from left anterior descending artery (LAD) territory by following parameters: summed stress score (SSS), summed rest scores (SRS), summed difference score (SDS, was calculated as the difference between SSS and SRS), stress TPD (STPD), rest TPD (RTPD), TPD difference (DTPD, was calculated as the difference between STPD and RTPD) [24, 25, 26].

\section{Statistical analysis}

The distribution of continuous variables was checked by using the Shapiro-Wilk's W-test. Normally distributed continuous variables were presented as the mean \pm standard deviation and not normally distributed parameters were shown as the median and interquartile range (Q25, Q75). Categorical variables were presented as numbers and percentages. Group comparisons were analyzed with Student ttest or the Mann-Whitney U-test for continuous variables, and the $\chi 2$ or Fisher's exact test for categorical 
variables. The Spearman test was used to estimate the correlation coefficient between quantitative variables. The receiver-operating-characteristic (ROC) curve analysis was performed to evaluate the sensitivity and specificity of tests. Areas under the ROC curves were compared using the DeLong method. A value of $p<0.05$ was considered statistically significant. All analyses were performed using SPSS statistical software 19.0 (SPSS Inc., Chicago, IL, USA) and MedCalc version 17.4 (MedCalc Software, Mariakerke, Belgium).

\section{Results}

\section{Clinical Outcomes}

The characteristics of the study groups are presented in Table 1. In 12 cases intra-aortic balloon counterpulsation was used intraoperatively or in the early postoperative period for circulatory assistance. The early mortality rate (within 30 days after surgery) was $11 \%$ (5/47). The causes of early death were associated with acute heart failure in four cases and gastrointestinal bleeding in one case.

\section{Coronary angiography results}

The stenosis of the main left coronary artery ranged from 40 to $75 \%$ was found in 8 cases. There were chronically totally occluded (CTO) left descending artery in 20 cases (42.6\%), more than $75 \%$ stenosis in 21 cases $(44,7 \%)$, and less than $70 \%$ in 6 cases $(12.7 \%)$. There were the following numbers of cases with stenosis in range 30 to 100 percentage: median artery in 4 cases, a diagonal branch of LAD in 16 cases, left circumflex artery in 9 cases, left marginal artery in 28 cases, a right coronary artery in 24 cases, a posterior descending artery in 11 cases.

\section{The results of CABG}

There were two cases of LIMA to LAD graft only, two grafts in 13 cases, three grafts in 24 cases, and more than three in the last 8 cases.

All 47 patients with LIMA to LAD grafts were divided into two groups: with graft satisfactory patency (Group 1, $n=40,85 \%$ ) and not-satisfactory patency (Group 2, $n=7,15 \%$ ) by TTFMs (cut-off value for $\mathrm{Q}=$ $11.5 \mathrm{ml} / \mathrm{min})$.

\section{Transit-time flow measurement}

The blood flow characteristics in the grafts are presented in Table 2. Patient groups had a statistically significant difference in the pulsatory index $(\mathrm{PI})$. The $\mathrm{Q}$ had correlations with $\mathrm{PI}(\mathrm{r}=-0.613, p<0.001)$ and diastolic flow $(r=0.418, p=0.003)$ values.

Despite the presence of LAD CTO in 20 cases, we did not find a statistically significant difference in flow measurements between patients with and without LAD CTO, and further analysis was carried out without division on that basis. The flow characteristics were different in patients with and without surgical left ventricle reconstruction (Table 3 ). 


\section{SPECT myocardial perfusion imaging}

All patients had at least 3 segments with abnormal myocardial perfusion. We found differences for both global and LAD territory myocardial perfusion characteristics only on rest MPI but not on stress (Table 4). The representative examples of myocardial SPECT with satisfactory and not-satisfactory TTFM are presented in Fig. 1.

According to the ROC analysis, TPD (rest) was the best predictor of the LIMA to LAD graft satisfactory: area under curve $=0.771$, cut-off value $=26.85$ with $83.3 \%$ sensitivity and $78.1 \%$ specificity .

The correlations between LIMA to LAD graft flow TTFMs with global and LAD territory MPI parameters are presented in Fig. 2.

\section{Discussion}

The preoperative rest myocardial perfusion in LAD territory can predict unsatisfactory blood flow in LIMA grafts in ICMP patients. Perfusion defect estimated as global and LAD territory SRS and TPD by rest myocardial SPECT with the necessity of SVR reasoned by apex/anterior LV aneurysm may lead in ICMP patients to low transit time flow measurements in LIMA to LAD grafts and consequently to graft failure.

TTFM is influenced by many factors, including vasoconstriction, graft diameter, perfusion area, morphological myocardial state, myocardial viability, myocardial oxygen consumption, blood pressure, and coronary vascular resistance [5]. Some of them will appear during or after the operation, but another part exists before the operation. The assessment of rest myocardial perfusion by SPECT before operation allows a surgeon to avoid a coronary vessel for LIMA graft destination with a high risk of graft occlusion via vessel territory TPD more than 26.85 .

\section{TTFM and graft patency}

Despite all coronary grafts occlude in no less than in 30\% by 1 year [27] it strongly depends on graft type. Thus, there were only 7\% in H. Oshima's et al (2016) and 6.5\% in P. Lehnert et al (2014) studies of failed artery grafts [1]. However, it was $15 \%$ of LIMA grafts with mean flow less than $11.5 \mathrm{ml} / \mathrm{min}$ in our investigation which can be explained with worse preoperative conditions of ICMP patients.

The low TTFM characteristics are correlated with the risk of LIMA graft failure at the one-year angiographic follow-up [13]. Even in the earliest studies, the pulsatory index was shown as a promising parameter (while there was no Q) it turned out that a significant difference was shown in Q between patent and non-patent grafts, but not in pulsatory index or diastolic flow [28].

According to the literature, the $Q$ for non-satisfactory arterial grafts is less than 10 to $20 \mathrm{ml} / \mathrm{min}[5,29]$. Lehnert et al defined the Q threshold of IMAs as $20 \mathrm{ml} / \mathrm{min}$ and Honda et al and G. Di Giammarco et al pointed out that reduced $Q<15 \mathrm{ml} / \mathrm{min}$ was found in non-satisfactory IMA grafts $[11,13,30]$. We 
considered a graft satisfactory with a normal waveform of blood flow, diastolic-dominant blood filling, and a mean flow value greater than $11.5 \mathrm{ml} / \mathrm{min}$.

A PI value may be elevated in very long arterial conduits because resistance to blood flow is determined by, among other factors, vessel length and increase PI associated with worse outcome [16, 31]. However, some authors supposed that PI is clinically irrelevant [22]. In our data PI value was irritated in patients with not-satisfactory patency of LIMA to LAD grafts.

\section{The TTFM measurements basis}

According to literature, TTFM is a quick and reproducible intraoperative method with the prognostic ability of graft potency in 5 years of the postoperative period. However, few investigators have written about reasons for low Q or high PI and possibilities of their prediction $[5,14,30]$. It is known that the technical problems in grafts can lead to changes in TTFM. This means it can be used for the detection of technical errors, but technical errors are only one source from many others of TTFM values shifts [32].

\section{Blood vessels}

The condition of the blood vessels, including graft state, obviously, has an impact on the graft flow. The measure in proximal graft end can be viewed as a sum of the graft capacitive flow and flow that passes through the distal anastomosis and the last one depends on many factors, such as graft destination, coronary artery stenosis, collateral, and competitive flows and sequential grafts [22]. The TTFM measurements are independent of graft diameter [33].

There is no direct study about graft destination impact to TTFMs, probably, because it is impossible to isolate this factor from others. However, Y. Tokuda et al (2007) declare different cut-off value of mean graft flow for different coronary arteries ( $15 \mathrm{~mL} / \mathrm{min}$ for LCA and $20 \mathrm{~mL} / \mathrm{min}$ for RCA) that indirectly suggest the presence impact of the destination artery [34].

$\mathrm{K}$. Honda et al showed that graft flow increases and pulsatory index decreases with progressing of native coronary stenosis [30]. Niclauss L in his review (2017) mentioned a correlation between blood flow and the severity of artery stenosis [16]. However, our data suggest that the CTO of the destination artery doesn't have an impact on TTFM in grafts and the low mean bypass graft flow and high pulsatory index measured by the TTFM are not specific for anastomotic stenosis. These blood flow characteristics might be explained with the competitive flow and poor coronary run-off [22].

Other vessel conditions poorly studied: Verhoye et al (2007) showed evidence and type of relationship of collateral blood flow between left coronary artery bypass grafts and chronically occluded right coronary artery in patients with triple vessel disease [35], but the influence of collateral circulation on graft patency is still not well known [1]; there is some evidence that competitive blood flow has a strong impact on graft's satisfactory in the literature [36]. The competition of blood flows can occur in the case of arterial $Y$ grafting directed to different coronary territories with unbalances stenosis [11]. The above data was a reason for excluding any sequential grafts from the analysis in this study. 
Ischemia

The current science knowledge lacks information about the impact of transitory ischemia on any TTFM measurements after CABG. H. Oshima et al (2016) wrote that they had assessed patients using preoperative SPECT but did not describe their results in the context of TTFM prediction [1]. In other literature, SPECT of myocardial perfusion is used for assessing coronary artery bypass graft disease state post CABG, but it didn't use for any associative analysis [37-42]. In our data, the preoperative myocardial perfusion SPECT characteristics related to ischemia in graft's destination coronary artery pool didn't affect Q, PI, or DF.

\section{Myocardial scar and SVR}

Some previous studies, including T. Murashita et al (2003), tried to use Gate-SPECT to improve the prediction of regional functional recovery after CBPG. They demonstrated that perfusion uptake of more than $50 \%$ was an indicator of functional recovery after revascularization [43]. We used the 17-segment model to estimate myocardium perfusion and had only SRS and TPD for LAD territory. The perfusion uptake was calculated by software for each segment and cannot be evaluated for LAD territory. That did impossible to compare our data in a clear manner, nevertheless, our cut-off point for LAD territory RS before the operation is $15.5 / 28(55.4 \%)$ and it is very close to the T. Murashita data.

Moreover, the perfusion uptake less than $50 \%$ in rest myocardial perfusion SPECT test is evidence of scar presenting in this area, which had to be in ICMP patients with LV aneurysm. The LV aneurysms in ICMP patients were fixed through SVR during the complex surgical treatment. In our study, Q was less and PI higher in IMA to LAD grafts in patients with SVR because of apex/anterior LV aneurysm what can be related to the part collateral circulation reduction or worse morphological myocardium condition before operation. In favor of the second theory, the patients with SVR had worse all myocardial perfusion characteristics except global and LAD territory SDS before the operation.

According to our study, the most suitable criteria for TTFM prediction in LIMA to LAD grafts in ICMP patients is total perfusion deficit (TPD) computed for vascular territory. The parameter, which combines pixel-based defect extent and severity, estimates an overall magnitude of hypoperfusion [44]. TPD more than $14 \%$ is significantly abnormal results [45] and our data suggests that TPD in LAD territory more than $26.85 \%$ is a criterion for the prediction of early LIMA graft failure in patients with ischemic cardiomyopathy.

\section{Study limitations}

The present study has a few limitations: there was a relatively small number of patients with non-potent grafts; the wall motion in the LAD territory was not assessed and we used cut-off value for mean conduit flow revealed by $\mathrm{H}$. Oshima et al (2016), despite an ongoing dispute about the cut-point values in modern literature. 


\section{Conclusion}

We must agree with T. Kieser et al, who determined that the use of TTFM is only half of the picture [4]. TTFM is a reliable tool for preventing technical mistakes during CABG, however, its use is limited by variability in presurgical myocardial condition. The TPD in LAD territory by preoperative myocardial rest perfusion SPECT can predict early graft failure in LIMA to LAD grafts in patients with ischemic cardiomyopathy, indicating that SPECT imaging can reduce risks of most reliable IMA graft failures.

\section{Declarations}

\section{Acknowledgments:}

No potential conflicts of interest were disclosed.

\section{Statements and Declarations}

The authors declare that no funds, grants, or other support were received during the preparation of this manuscript.

The authors have no relevant financial or non-financial interests to disclose.

All authors contributed to the study conception and design. Material preparation, data collection and analysis were performed by Sergey S. Gutor, Sergey L. Andreev, Vladimir V. Shipulin, Andrey V. Mochula, Vasiliy V. Zatolokin, Andrey S. Pryahin, Vladimir M. Shipulin, Boris N. Kozlov, and Konstantin V. Zavadovsky. The first draft of the manuscript was written by Sergey S. Gutor and all authors mentioned above. All authors read and approved the final manuscript

The study was approved by the Local Ethical Committee (Committee on Biomedical Ethics, Cardiology Research Institute; Approval Number: 178) and conformed to the Declaration of Helsinki on Human Research. Written informed consent was obtained from each patient after explanation of the protocol, its aims, and potential risks.

\section{References}

1. Oshima H, Tokuda Y, Araki Y, Ishii H, Murohara T, Ozaki Y, Usui A (2016) Predictors of early graft failure after coronary artery bypass grafting for chronic total occlusion. Interact Cardiovasc Thorac Surg 23:142-149

2. Velazquez EJ, Lee KL, Deja MA et al (2011) Coronary-artery bypass surgery in patients with left ventricular dysfunction. N Engl J Med 364:1607-1616

3. Amin S, Werner RS, Madsen PL, Krasopoulos G, Taggart DP (2018) Intraoperative Bypass Graft Flow Measurement With Transit Time Flowmetry: A Clinical Assessment. Ann Thorac Surg 106:532-538 
4. Kieser TM, Taggart DP (2018) Current status of intra-operative graft assessment: Should it be the standard of care for coronary artery bypass graft surgery? J Card Surg 33:219-228

5. Takami Y, Takagi Y (2018) Roles of Transit-Time Flow Measurement for Coronary Artery Bypass Surgery. Thorac Cardiovasc Surg 66:426-433

6. Walpoth BH, Mohadjer A, Gersbach P, Rogulenko R, Walpoth BN, Althaus U (1996) Intraoperative internal mammary artery transit-time flow measurements: comparative evaluation of two surgical pedicle preparation techniques. Eur J Cardiothorac Surg 10:1064-1068 discussion 1069-1070

7. Wu S-J, Li Y-C, Shi Z-W, Lin Z-H, Rao Z-H, Tai S-C, Chu M-P, Li L, Lin J-F (2017) Alteration of Cholinergic Anti-Inflammatory Pathway in Rat With Ischemic Cardiomyopathy-Modified Electrophysiological Function of Heart. J Am Heart Assoc. https://doi.org/10.1161/JAHA.117.006510

8. Strecker T, Rösch J, Weyand M, Agaimy A (2014) Pathological findings in cardiac apex removed during implantation of left ventricular assist devices (LVAD) are non-specific: 13-year-experience at a German Heart Center. Int J Clin Exp Pathol 7:5549-5556

9. Hol PK, Fosse E, Mork BE et al (2001) Graft control by transit time flow measurement and intraoperative angiography in coronary artery bypass surgery. Heart Surg Forum 4:254-257 discussion 257-258

10. Neumann F-J, Sousa-Uva M, Ahlsson A et al (2019) 2018 ESC/EACTS Guidelines on myocardial revascularization. Eur Heart J 40:87-165

11. Di Giammarco G, Pano M, Cirmeni S, Pelini P, Vitolla G, Di Mauro M (2006) Predictive value of intraoperative transit-time flow measurement for short-term graft patency in coronary surgery. $\mathrm{J}$ Thorac Cardiovasc Surg 132:468-474

12. Task Force on Myocardial Revascularization of the European Society of Cardiology (ESC) and the European Association for Cardio-Thoracic Surgery (EACTS), European Association for Percutaneous Cardiovascular Interventions (EAPCI), Wijns W et al (2010) Guidelines on myocardial revascularization. Eur Heart J 31:2501-2555

13. Lehnert P, Møller CH, Damgaard S, Gerds TA, Steinbrüchel DA (2015) Transit-time flow measurement as a predictor of coronary bypass graft failure at one year angiographic follow-up. J Card Surg $30: 47-52$

14. Su P, Gu S, Liu Y, Zhang X, Yan J, An X, Gao J, Xin Y, Zhou J (2018) Off-Pump Coronary Artery Bypass Grafting with Mini-Sternotomy in the Treatment of Triple-Vessel Coronary Artery Disease. Int Heart J 59:474-481

15. Yu Y, Zhang F, Gao M-X, Li H-T, Li J-X, Song W, Huang X-S, Gu C-X (2013) The application of intraoperative transit time flow measurement to accurately assess anastomotic quality in sequential vein grafting. Interact Cardiovasc Thorac Surg 17:938-943

16. Niclauss $L$ (2017) Techniques and standards in intraoperative graft verification by transit time flow measurement after coronary artery bypass graft surgery: a critical review. Eur J Cardiothorac Surg $51: 26-33$ 
17. Felker GM, Shaw LK, O'Connor CM (2002) A standardized definition of ischemic cardiomyopathy for use in clinical research. J Am Coll Cardiol 39:210-218

18. Kirklin/Barratt-Boyes Cardiac Surgery - 9781416063919 | US Elsevier Health Bookshop. https://www.us.elsevierhealth.com/kirklinbarratt-boyes-cardiac-surgery9781416063919.html\#panel1. Accessed 19 Jun 2019

19. Menicanti L, Di Donato M (2002) The Dor procedure: what has changed after fifteen years of clinical practice? J Thorac Cardiovasc Surg 124:886-890

20. Cooley DA (1989) Ventricular endoaneurysmorrhaphy: a simplified repair for extensive postinfarction aneurysm. J Card Surg 4:200-205

21. Judkins MP (1967) Selective coronary arteriography. I. A percutaneous transfemoral technic. Radiology 89:815-824

22. Jelenc M, Jelenc B, Klokočovnik T, Lakič N, Geršak B, Kneževic I (2014) Understanding coronary artery bypass transit time flow curves: role of bypass graft compliance. Interact Cardiovasc Thorac Surg 18:164-168

23. Henzlova MJ, Duvall WL, Einstein AJ, Travin MI, Verberne HJ (2016) ASNC imaging guidelines for SPECT nuclear cardiology procedures: Stress, protocols, and tracers. J NuclCardiol 23:606-639

24. Cerqueira MD, Weissman NJ, Dilsizian V et al (2002) Standardized myocardial segmentation and nomenclature for tomographic imaging of the heart. A statement for healthcare professionals from the Cardiac Imaging Committee of the Council on Clinical Cardiology of the American Heart Association. Circulation 105:539-542

25. Germano G, Kavanagh PB, Waechter P, Areeda J, Van Kriekinge S, Sharir T, Lewin HC, Berman DS (2000) A new algorithm for the quantitation of myocardial perfusion SPECT. I: technical principles and reproducibility. J Nucl Med 41:712-719

26. Ficaro EP, Lee BC, Kritzman JN, Corbett JR (2007) Corridor4DM: the Michigan method for quantitative nuclear cardiology. J NuclCardiol 14:455-465

27. Jokinen JJ, Werkkala K, Vainikka T, Peräkylä T, Simpanen J, Ihlberg L (2011) Clinical value of intraoperative transit-time flow measurement for coronary artery bypass grafting: a prospective angiography-controlled study. Eur J Cardiothorac Surg 39:918-923

28. Walker PF, Daniel WT, Moss E, Thourani VH, Kilgo P, Liberman HA, Devireddy C, Guyton RA, Puskas JD, Halkos ME (2013) The accuracy of transit time flow measurement in predicting graft patency after coronary artery bypass grafting. Innovations (Phila) 8:416-419

29. Di Giammarco G, Rabozzi R (2010) Can transit-time flow measurement improve graft patency and clinical outcome in patients undergoing coronary artery bypass grafting? Interact Cardiovasc Thorac Surg 11:635-640

30. Honda K, Okamura Y, Nishimura Y, Uchita S, Yuzaki M, Kaneko M, Yamamoto N, Kubo T, Akasaka T (2015) Graft flow assessment using a transit time flow meter in fractional flow reserve-guided coronary artery bypass surgery. J Thorac Cardiovasc Surg 149:1622-1628 
31. Kieser TM, Rose S, Kowalewski R, Belenkie I (2010) Transit-time flow predicts outcomes in coronary artery bypass graft patients: a series of 1000 consecutive arterial grafts. Eur J Cardiothorac Surg 38:155-162

32. Leong DKH, Ashok V, Nishkantha A, Shan YH, Sim EKW (2005) Transit-time flow measurement is essential in coronary artery bypass grafting. Ann Thorac Surg 79:854-857 discussion 857-858

33. Matre K, Birkeland S, Hessevik I, Segadal L (1994) Comparison of transit-time and Doppler ultrasound methods for measurement of flow in aortocoronary bypass grafts during cardiac surgery. Thorac Cardiovasc Surg 42:170-174

34. Tokuda $\mathrm{Y}$, Song M-H, Ueda Y, Usui A, Akita T (2007) Predicting early coronary artery bypass graft failure by intraoperative transit time flow measurement. Ann Thorac Surg 84:1928-1933

35. Verhoye J-P, Abouliatim I, Drochon A, de Latour B, Leclercq C, Leguerrier A, Corbineau H (2007) Collateral blood flow between left coronary artery bypass grafts and chronically occluded right coronary circulation in patients with triple vessel disease. Observations during complete revascularisation of beating hearts. Eur J Cardiothorac Surg 31:49-54

36. Rossi M, Jiritano F, Malta E, Renzulli A (2012) Competitive flow between a vein and an arterial graft at transit-time flow measurement. Interact Cardiovasc Thorac Surg 15:288-289

37. Pinarli null, Ersek null (1998) Gürsürer null, Aksoy null, Yavuz null, Assessment of Graft Patency Rate After Coronary Artery Bypass Surgery by Exercise TL-201 Single Photon Emission Computed Tomography. Int J Angiol 7:313-316

38. Taki J, Ichikawa A, Nakajima K, Kawasuji M, Tonami N (1997) Comparison of flow capacities of arterial and venous grafts for coronary artery bypass grafting: evaluation with exercise thallium-201 single-photon emission tomography. Eur J Nucl Med 24:1487-1493

39. Yada T, Futagami Y, Koyama T, Iwata J, Konishi T, Hamada M, Nakano T, Takezawa H, Yuasa H, Kusagawa M (1988) [Graft patency and myocardial viability after aorto-coronary bypass surgery evaluated by exercise 201T1 myocardial SPECT]. J Cardiol 18:299-306

40. Kureshi SA, Tamaki N, Yonekura Y, Koide H, Konishi Y, Ban T, Konishi J, Torizuka K (1989) Value of stress thallium-201 emission tomography for predicting improvement after coronary bypass grafting and assessing graft patency. Jpn Heart J 30:287-299

41. Lakkis NM, Mahmarian JJ, Verani MS (1995) Exercise thallium-201 single photon emission computed tomography for evaluation of coronary artery bypass graft patency. Am J Cardiol 76:107111

42. Al Aloul B, Mbai M, Adabag S et al (2012) Utility of nuclear stress imaging for detecting coronary artery bypass graft disease. BMC Cardiovasc Disord 12:62

43. Murashita T, Makino Y, Kamikubo Y, Yasuda K, Mabuchi M, Tamaki N (2003) Quantitative gated myocardial perfusion single photon emission computed tomography improves the prediction of regional functional recovery in akinetic areas after coronary bypass surgery: useful tool for evaluation of myocardial viability. J Thorac Cardiovasc Surg 126:1328-1334 
44. Slomka P, Xu Y, Berman D, Germano G (2012) Quantitative Analysis of Perfusion Studies: Strengths and Pitfalls. J NuclCardiol 19:338-346

45. Czaja M, Wygoda Z, Duszańska A, Szczerba D, Głowacki J, Gąsior M, Wasilewski JP (2017) Interpreting myocardial perfusion scintigraphy using single-photon emission computed tomography. Part 1. Kardiochir Torakochirurgia Pol 14:192-199

\section{Tables}

Table 1 The clinical characteristics of study groups 


\begin{tabular}{|c|c|c|c|}
\hline Groups & $\mathrm{n}$ & Ages (years) & Comparison \\
\hline Group 1, (Q>11,5 ml/min) & $\mathrm{n}=40$ & $61.4 \pm 4.9$ & \multirow[t]{2}{*}{$t=0.73, p=0.48$} \\
\hline Group 2, (Q $\leq 11,5 \mathrm{ml} / \mathrm{min})$ & $\mathrm{n}=7$ & $59.4 \pm 6.2$ & \\
\hline Diabetes Mellitus ( $2^{\text {nd }}$ type) & Group 1 & Group 2 & \\
\hline Prevalence, n (\%) & $\mathrm{n}=8(20 \%)$ & $\mathrm{n}=1(14 \%)$ & $p>0.05$ \\
\hline NYHA class, n (\%) & Group 1 & Group 2 & \\
\hline I & $\mathrm{n}=1(3 \%)$ & $\mathrm{n}=1(14 \%)$ & \multirow[t]{4}{*}{$p>0.05$} \\
\hline ॥ & $\mathrm{n}=22(55 \%)$ & $\mathrm{n}=2(28 \%)$ & \\
\hline III & $\mathrm{n}=14(35 \%)$ & $\mathrm{n}=4(58 \%)$ & \\
\hline IV & $\mathrm{n}=3(7 \%)$ & $\mathrm{n}=0(\%)$ & \\
\hline CCS angina class, $\mathrm{n}(\%)$ & Group 1 & Group 2 & \\
\hline I & $\mathrm{n}=1(3 \%)$ & $\mathrm{n}=1(14 \%)$ & \multirow[t]{4}{*}{$p>0.05$} \\
\hline II & $\mathrm{n}=11(27 \%)$ & $\mathrm{n}=4(58 \%)$ & \\
\hline III & $\mathrm{n}=27(67 \%)$ & $\mathrm{n}=2(28 \%)$ & \\
\hline IV & $\mathrm{n}=1(3 \%)$ & $\mathrm{n}=0(\%)$ & \\
\hline The operation type, $\mathrm{n}(\%)$ & Group 1 & Group 2 & \\
\hline CABG & $n=25(63 \%)$ & $\mathrm{n}=2(28 \%)$ & \multirow[t]{3}{*}{$p>0.05$} \\
\hline CABG+SVR (D. Cooley) & $\mathrm{n}=7(17 \%)$ & $\mathrm{n}=1(14 \%)$ & \\
\hline CABG+SVR (L. Menicanti) & $\mathrm{n}=8(20 \%)$ & $\mathrm{n}=4(58 \%)$ & \\
\hline Ultrasound data & Group 1 & Group 2 & \\
\hline LV ESV & $136(120,184)$ & $173(154,182)$ & $U=199, p=0.08$ \\
\hline LV EDV & $201(189,251)$ & $249(223,265)$ & $U=204, p=0.06$ \\
\hline LV EF (\%) & $31.1 \pm 6.1$ & $30.29 \pm 5.5$ & $t=0.37, p=0.72$ \\
\hline Others & Group 1 & Group 2 & \\
\hline Body-mass index $\left(\mathrm{kg} / \mathrm{m}^{2}\right)$ & $27.4(25.3,30.9)$ & $28.6(26.5,31.1)$ & $U=117, p=0.85$ \\
\hline EuroSCORE & $4.5 \pm 2.1$ & $3.9 \pm 2.1$ & $U=104, p=0.36$ \\
\hline
\end{tabular}

Table 2 Results of intraoperative transit-time flow measurements in LIMA to LAD grafts 


\begin{tabular}{llll} 
Transit-time flow measurements & Group 1 & Group 2 & Comparison \\
\hline $\mathrm{Q}(\mathrm{ml} / \mathrm{min})$ & $43(23,61.8)$ & $9(5.5,10)$ & N.A. \\
\hline $\mathrm{PI}^{*}$ & $3.0(2.4,3.7)$ & $7.1(4.5,10.8)$ & $\mathrm{U}=240.5, \mathrm{p}=0.003$ \\
\hline $\mathrm{DF}(\%)$ & $69.5(58.5,74.5)$ & $60(37.5,66)$ & $\mathrm{U}=89, \mathrm{p}=0.131$
\end{tabular}

* - a statistically significant difference, $p<0.05$.

Table 3 The impact of CTO and SVR on results of intraoperative transit-time flow measurements in LIMA to LAD grafts

\begin{tabular}{llll} 
Transit-time flow measurements & with LAD CTO & without LAD CTO & Comparison \\
\hline $\mathrm{Q}(\mathrm{ml} / \mathrm{min})$ & $36.5(13.5,53.5)$ & $30(16,57)$ & $\mathrm{U}=287, \mathrm{p}=0.723$ \\
\hline $\mathrm{PI}$ & $3.6(2.8,5.3)$ & $3.0(2.4,4.0)$ & $\mathrm{U}=227.5, \mathrm{p}=0.366$ \\
\hline $\mathrm{DF}(\%)$ & $67.5(56.8,73.3)$ & $69(62,76)$ & $\mathrm{U}=292, \mathrm{p}=0.643$ \\
\hline Transit-time flow measurements & with SVR & without SVR & Comparison \\
\hline $\mathrm{Q}(\mathrm{ml} / \mathrm{min})^{\star}$ & $17(11.5,40.8)$ & $45(25,67.5)$ & $\mathrm{U}=386.5, \mathrm{p}=0.013$ \\
\hline $\mathrm{PI}$ & $3.9(3.2,7.4)$ & $2.9(2.3,3.6)$ & $\mathrm{U}=135.5, \mathrm{p}=0.004$ \\
\hline $\mathrm{DF}(\%)^{\star}$ & $63.5(44.5,70.8)$ & $72(66.5,77)$ & $\mathrm{U}=372, \mathrm{p}=0.029$
\end{tabular}

* - a statistically significant difference, $p<0.05$.

Table 4 Results of SPECT MPI 


\begin{tabular}{llll} 
SPECT & Group 1 & Group 2 & Comparison \\
\hline Global SPECT estimates & & & \\
\hline SSS & $27.3 \pm 8.3$ & $32.7 \pm 5.7$ & $\mathrm{t}=2.2, \mathrm{p}=0.051$ \\
\hline TPD (stress) & $38.7 \pm 10.4$ & $45.7 \pm 8.5$ & $\mathrm{t}=2.0, \mathrm{p}=0.081$ \\
\hline SRS* & $20.7 \pm 7.5$ & $28.8 \pm 4.3$ & $\mathrm{t}=3.7, \mathrm{p}=0.003$ \\
\hline TPD (rest)* & $32.0 \pm 11.4$ & $43.7 \pm 8.1$ & $\mathrm{t}=3.0, \mathrm{p}=0.014$ \\
\hline SDS & $4(2,7)$ & $3(3,4.5)$ & $\mathrm{U}=93.5, \mathrm{p}=0.936$ \\
\hline LAD territory SPECT estimates & & & \\
\hline SS & $12.7 \pm 6.0$ & $15.3 \pm 7.7$ & $\mathrm{t}=0.9, \mathrm{p}=0,421$ \\
\hline TPD (stress) & $21.4 \pm 8.9$ & $25.1 \pm 11.7$ & $\mathrm{t}=0.8, \mathrm{p}=0.449$ \\
\hline RS & $10.8 \pm 5.4$ & $16.2 \pm 5.1$ & $\mathrm{t}=2.3, \mathrm{p}=0.052$ \\
\hline TPD (rest)* & $19.7 \pm 9.6$ & $28.4 \pm 7.7$ & $\mathrm{t}=2.4, \mathrm{p}=0.040$ \\
\hline DS & $0(0,2)$ & $1(0.3,1.8)$ & $\mathrm{U}=111.0, \mathrm{p}=0.532$
\end{tabular}

Figures 


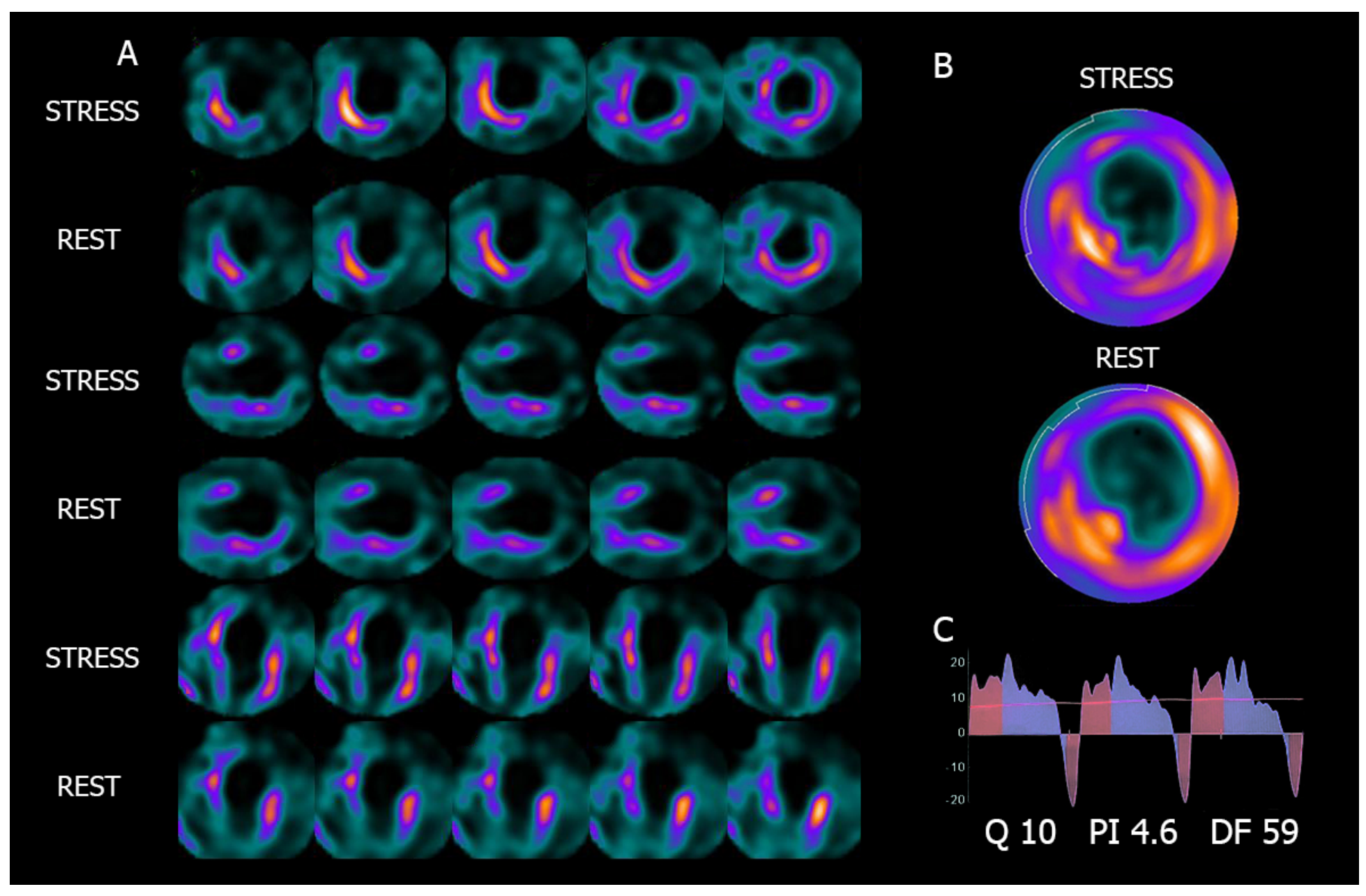

Figure 1

The myocardial perfusion stress-rest SPECT $(a, b)$ in ICMP patients with non-satisfactory $(Q<11.5$, left) and satisfactory ( $Q>11.5$, right) flow in IMA grafts during CABG (c) 


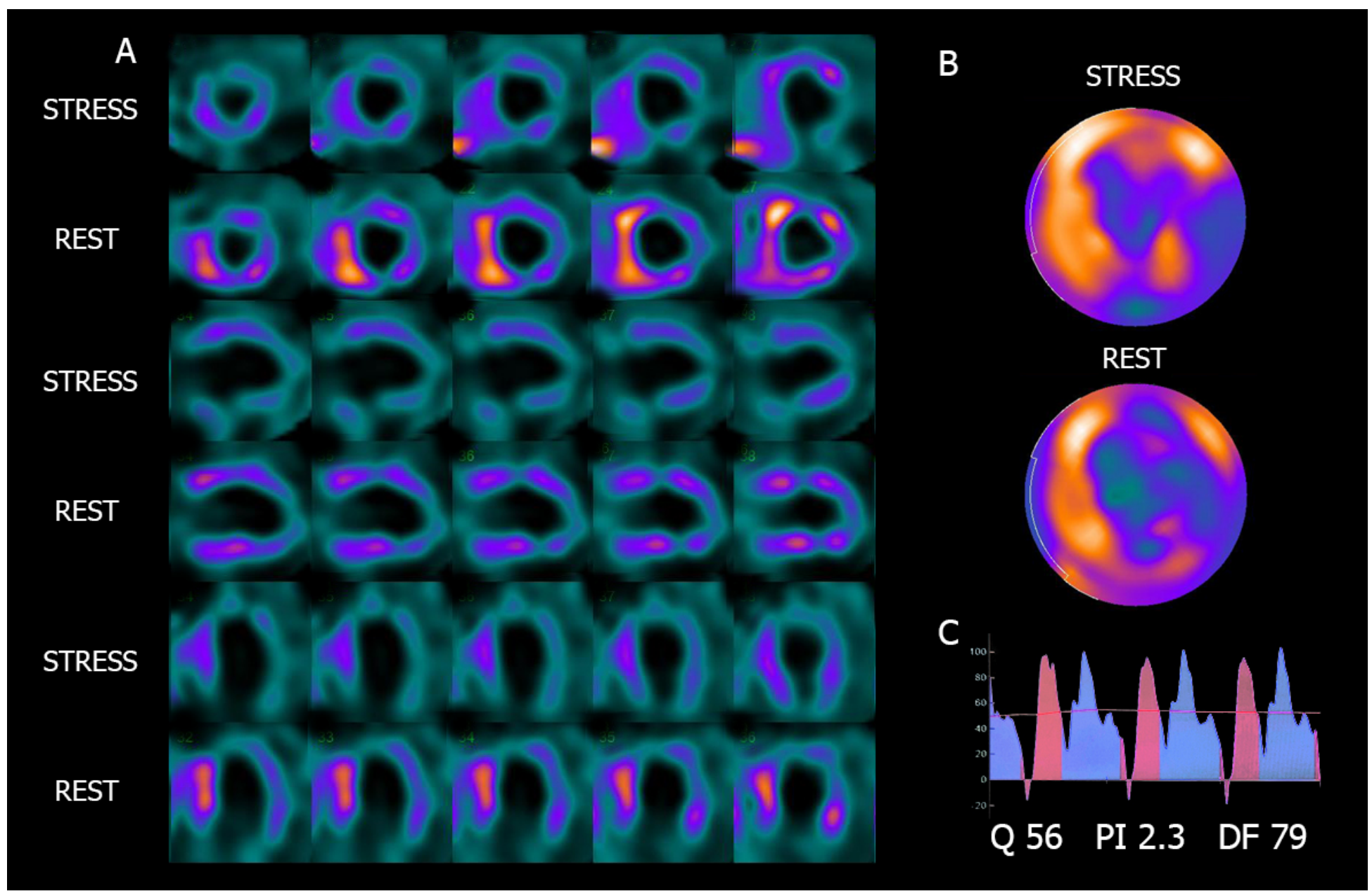

Figure 2

Correlations between TTFM and MPI estimators. * - statistically significant correlation, $p<0.05$ 